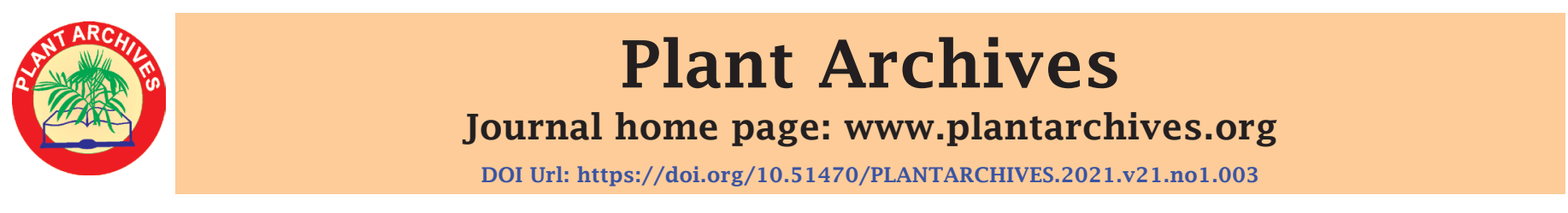

\title{
PLANT DIVERSITY OF KANSARI MAVLI SACRED GROVE OF SONGADH FOREST RANGE IN TAPI DISTRICT, GUJARAT, INDIA
}

\section{Arpit D. Gamit* and Bharat B. Maitreya}

Department of Botany, Bioinformatics and Climate Change Impact Management, University School of Science, Gujarat University, Ahmedabad, Gujarat, India

*E-mail: arpitgmt008@gmail.com

(Date of Receiving-07-09-2020; Date of Acceptance-28-11-2020)

Sacred groves are forest patches being conserved by tribal people. Sacred groves are closely associated with religious practices,
social duties and taboos. The present study attempts show a key role play to conserve plant species by Kansari Mavli sacred
grove. The sacred grove is situated in songadh range of vyara division, Gujarat state. Due to excess use of forest resources for
ABSTRACT $\begin{aligned} & \text { timber, fodder and food, forest cover areas are rapidly decreasing. This forest patch has rich plant diversity and is protected by } \\ & \text { local people. Total } 149 \text { plant species are recorded, studied and collected }\end{aligned}$
Keywords: Plant diversity, Sacred grove, Songadh forest, Gujarat, India.

\section{INTRODUCTION}

Sacred groves are forest patches of rich vegetation traditionally been protected on the ground of religious beliefs and are dedicated to many gods, goddesses, holy spirits etc. As described by Vartak (1983), sacred groves are natural museums of living giant trees, treasure house of rare, endemic and endangered species, dispensary of medicinal plants, recreation centre for urban life, garden for botanists, gene bank of economic species, paradise for nature lovers and laboratory for environmentalists. They are considered as repository of local biodiversity

The sacred groves play an important role in ecosystem services by providing clean air, soil and water, conservation of flora and fauna, temperature control and conservation of traditional knowledge. Being a biotype in a rural landscape, the sacred groves performs a critical role in the maintenance of ecological and hydrological balance of an area, and also help to compensate for carbon emissions of polluting industries and thereby offers economic benefits to the communities besides other ecological benefits (Chandrashekara, 2011).

In India the sacred groves are found scattered all over the country especially in the Khasi and Jaintia hills of Northeastern Himalayas, Aravalli Hills, Central India and Western Ghats. They are known under different names in different states like Dev van in Himachal Pradesh, Oransin Rajasthan, Sarnasin Bihar, Lai Umangin Manipur, Ki Law Kyntangin Meghalaya, Devor Sarnain Madhya Pradesh, Deovani, Devrai or Devrahati in Maharashtra, Devarabana, Devarakadu or Pavitravana in Karnataka, Kavu or Kovilkadu in Tamil Nadu, and Kavuin Kerala (Muruganet al., 2008).

Several Sacred Groves are seen throughout

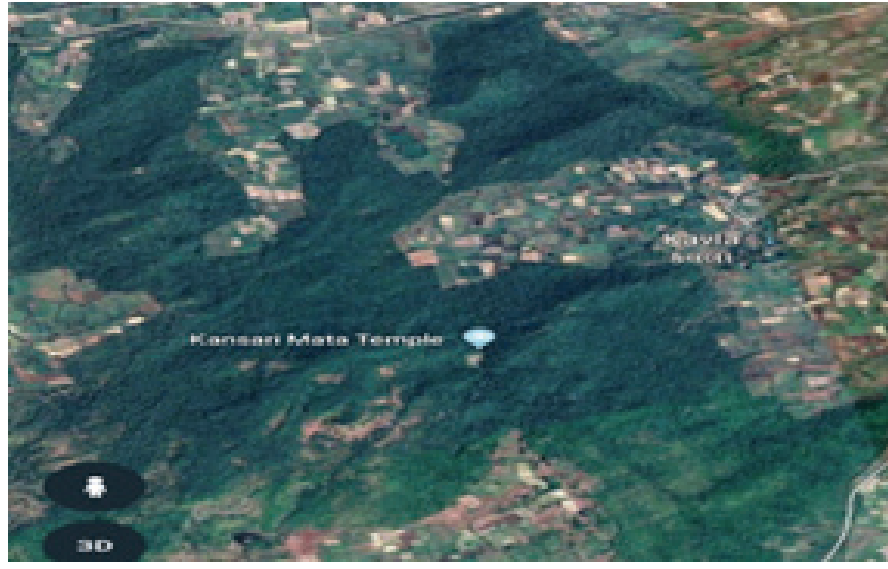

Fig, 1 Map showing Kansari Mavli Forest area
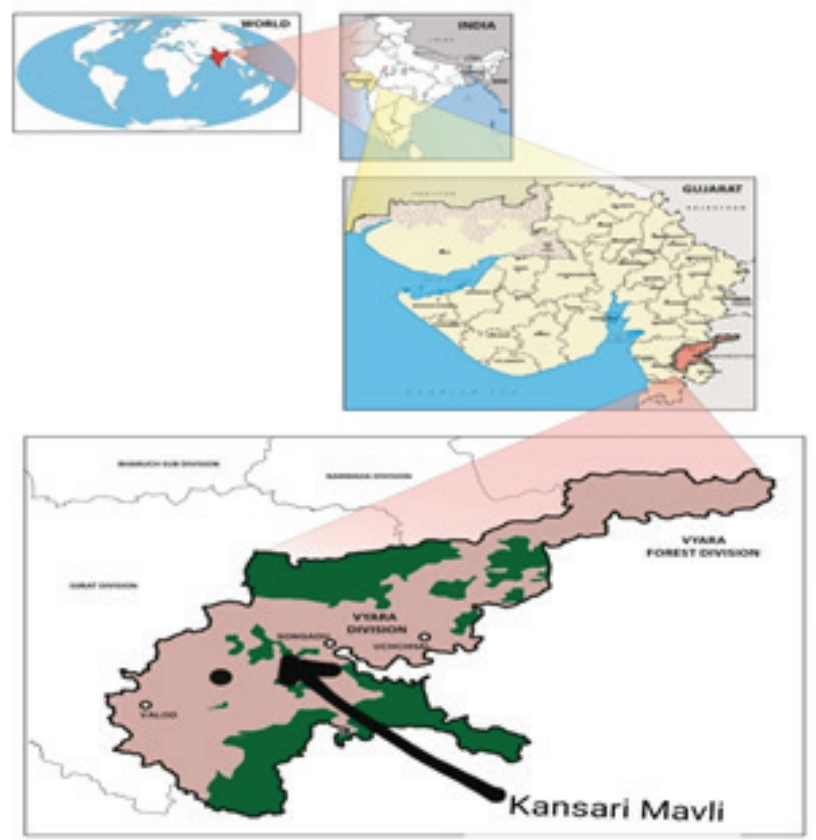

Fig. 2 Location map of Kansari Mavli Sacred Grove 


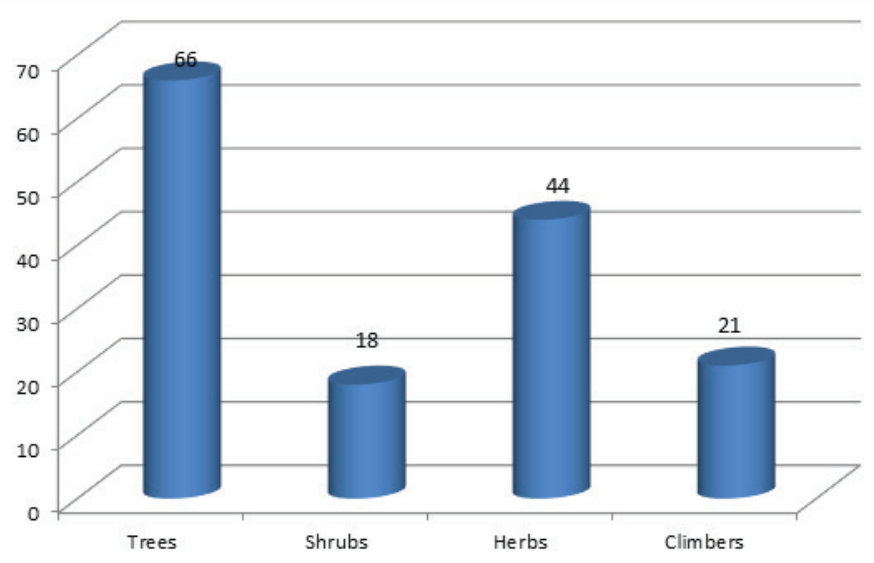

Fig. 3 Graph showing habit wise distribution of plant species

Gujarat in various forms. Sacred Groves are of much significant in indigenous people's religious and cultural practices. In Gujarat indigenous people inhabit in eastern forests belt. Over 42 Sacred Groves have been documented in Banaskantha, Sabarkantha, Dahod and Kutchh districts.

\section{MATERIALS AND METHODS}

Study Area: Kansarimavli is the goddess of grain which corresponds to the gods and goddesses of the settled tribal agriculturists. The sacred grove is located at the foot of a steep hill of highly forested area between the Nishana and Kavla villages of Tapi district. The study area is situated

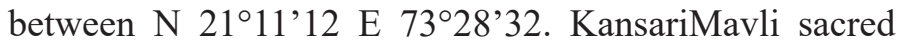
grove is one of the many sacred groves situated in Songadh forest range of Vyara division.Vyara Forest Division is located in South Gujarat, a region in Indian State of Gujarat. Vyara forest division covers mainly the eastern part of Surat district and major area in the western part of Tapi district. The study area is situated between latitude $20^{\circ} 46^{\prime} \mathrm{N}$ and $21^{\circ} 27^{\prime} \mathrm{N}$ and longitude $73^{\circ} 27^{\prime} \mathrm{E}$ and $73^{\circ} 59^{\prime}$ E. Forests are mostly confined to the eastern hilly region of the district. The Forest area of Vyara Forest Division is $907.60 \mathrm{~km}^{2}$ which covers $28.02 \%$ of total geographic area (*GFD report data 2016).

\section{METHODOLOGY}

Kansari Mavli sacred grove is in the Gamit tribal dominant Songadh forest range of vyara division. The study area was identified and frequently visited by help of local villagers. The GPS location was also been taken by Google Earth. The flowering plants were explored and collected during several field visits. The study was carried out during September 2017 to August 2019. Plant species were studied and identified with the help of local flora and taxonomy expert, photographs were also been captured of the sacred grove and flowering plants. The collected specimens were stored in a form of herbarium.

\section{RESULTS AND DISCUSSION}

Several plant species trees, shrubs, herbs and climbers have been protected by tribal people in this area. A total of 149 species of plants under 66 families and 121 genera including 66 species of trees, 18 species of shrubs, 44 species of herbs and 21 species of climbers are recorded. Fabaceae is the most dominant family having 13 genera and 14 species. In the present study, Kansari Mavli sacred grove has been documented of Songadh forest range in Vyara division. The sacred grove is dedicated to Kansari Mata which is goddess of grain. Mainly Gamit and Chaudhari tribes of surrounding areas offer grain seeds to her before monsoon in June months every year. The thanks giving ceremony is also been performed after the preparation of new

Fig, 1 Map showing Kansari Mavli Forest area crop.

\section{CONCLUSION}

The present study helps to understand the direct or indirect close relation between indigenous people and sacred groves. Therefore, has important to explore the plant wealth of the area and dependence of tribal on it. It also contributes to know the ecologicalsignificance of such sacred forests in order to preserve its rich diversity of flora and fauna.

\section{Photographs of Sacred Grove and plants}

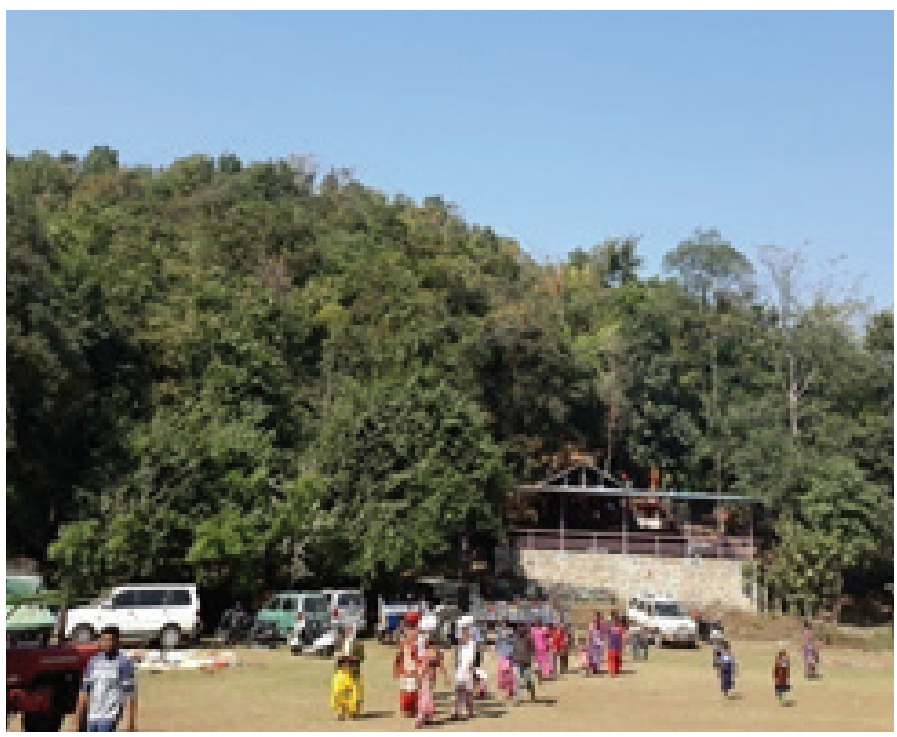

Kansari- Mata Sacred Grove

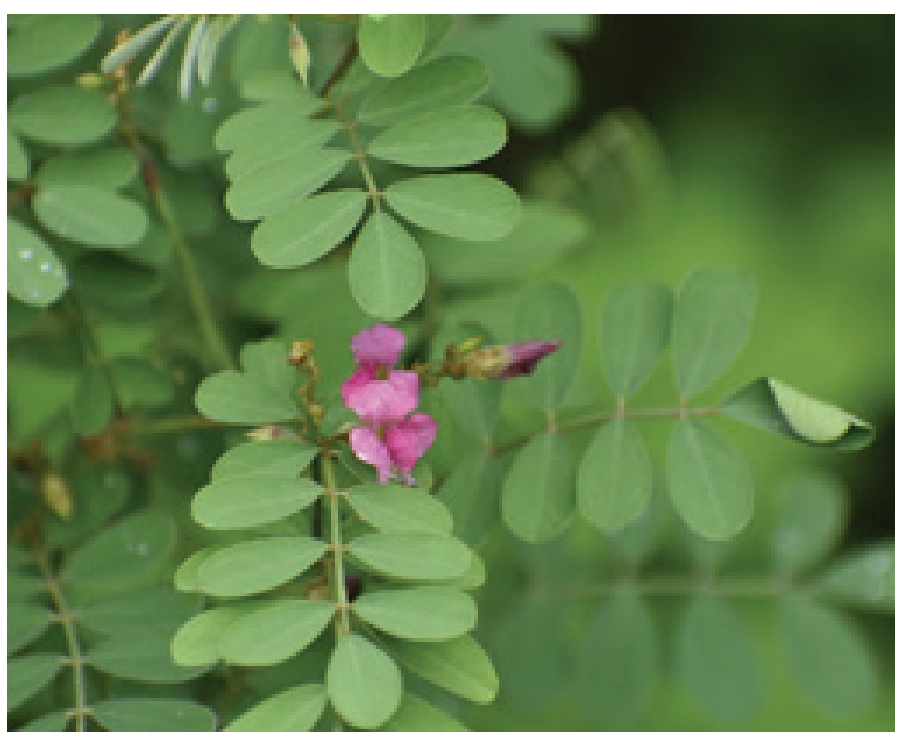

Tephrosia purpurea ( Linn.) Pers. 
Table: 1 Plant diversity of KansariMavli Sacred Grove of Songadh range

\begin{tabular}{|c|c|c|c|c|}
\hline Sr No & Scientific Name & Family & Common Name & Habit \\
\hline 1 & Rungia pectinata (Linn.) Nees & Acanthaceae & Khadselio & Herb \\
\hline 2 & Achyranthes aspera Linn. & Amaranthaceae & Anghedi & Herb \\
\hline 3 & Alternanthera paronychioides St. -Hill. & Amaranthaceae & & Herb \\
\hline 4 & Gomphrena celosioides Mart. & Amaranthaceae & & Herb \\
\hline 5 & Lannea coromandelica (Houtt.) Merr. & Anacardiaceae & Moyno,Modal & Tree \\
\hline 6 & Mangifera indica Linn. & Anacardiaceae & Amba, Mango & Tree \\
\hline 7 & Miliusato mentosa (Robx.) Sinclair & Annonaceae & Umbh & Tree \\
\hline 8 & Cryptolepis dubia (Burm.f.) M.R.Almeida & Apocynaceae & & Climber \\
\hline 9 & Holarrhena pubescens (Buch.-Ham.) Wall. & Apocynaceae & Kudo, Indrajav & Shrub \\
\hline 10 & Wrightia arborea (Dennst.) Mabberley & Apocynaceae & Dudhi & Tree \\
\hline 11 & Wrightia tinctoriaR.Br. & Apocynaceae & DudhKudi & Tree \\
\hline 12 & Colocasia esculenta & Araceae & Taro & Herb \\
\hline 13 & Calotropis procera (Ait.) R.Br. & Asclepiadaceae & Akado & Shrub \\
\hline 14 & Tridax procumbens Linn. & Asteraceae & Pardeshibhangro & Herb \\
\hline 15 & Balanites aegyptiaca (Linn.) Del. & Balatinaceae & Ingorio, Hingoriyo & Tree \\
\hline 16 & Impatiens balsamina Linn. & Balsaminaceae & & Herb \\
\hline 17 & Oroxylum indicum (Linn.) Vent. & Bignoniaceae & Tetu & Tree \\
\hline 18 & Tecomella undulata (Sm.) Seem. & Bignoniaceae & Ragatrohido & Tree \\
\hline 19 & Ceiba pentandra (Linn.) Gaertn. & Bombacaceae & & Tree \\
\hline 20 & Garuga pinnataRobx. & Burseraceae & Kakad & Tree \\
\hline 21 & Bauhinia racemosaLamk. & Caesalpiniaceae & Ashitro & Tree \\
\hline 22 & Caesalpinia bonduc (L.)Robx. & Caesalpiniaceae & Kantkaranj & Shrub \\
\hline 23 & Cassia fistula Linn. & Caesalpiniaceae & Garmado & Tree \\
\hline 24 & Cassia occidentalis Linn. & Caesalpiniaceae & Sundro & Shrub \\
\hline 25 & Cassia tora Linn. & Caesalpiniaceae & Kuvadio & Herb \\
\hline 26 & Delonix regia (Boj. ex Hk.) Raf. & Caesalpiniaceae & Gulmohar & Tree \\
\hline 27 & Tamarindus indica Linn. & Caesalpiniaceae & Amali & Tree \\
\hline 28 & Capparis decidua (Forssk.) Edg. & Capparaceae & Kerdo,Kera & Shrub \\
\hline 29 & Crateva tapia & Capparaceae & & Tree \\
\hline 30 & Cassine glauca (Rottb.) O. Ktze. & Celastraceae & Bhut zad & Tree \\
\hline 31 & Celastrus paniculatusWilld. & Celastraceae & Malkagani & Climber \\
\hline 32 & Maytenuse marginata (Willd.) Ding Hou & Celastraceae & Vickdo, Vico & Shrub \\
\hline 33 & Cleome viscosa Linn. & Cleomaceae & Pili-tilvan & Herb \\
\hline 34 & Anogeisus latifolia (Robx. ex DC.) Guill. & Combretaceae & Dhavdo & Tree \\
\hline 35 & Anogiessus pendula Edgew. & Combretaceae & Safed Dhav & Tree \\
\hline 36 & Terminalia crenulataRoth. & Combretaceae & Sadad & Tree \\
\hline 37 & Terminalia arjuna (Robx. ex DC.) Wight & Combretaceae & Arjun sadad & Tree \\
\hline 38 & Terminalia bellirica (Gaertn.) Robx. & Combretaceae & Baheda & Tree \\
\hline 39 & Commelina benghalensis Linn. & Commelinaceae & & Herb \\
\hline 40 & Commelina diffusaBurm.f. & Commelinaceae & & Herb \\
\hline 41 & Ipomoea aquaticaForsk. & Convolvulaceae & Nalinibhajil & Climber \\
\hline 42 & Ipomoea muricata (Linn.) Jacq. & Convolvulaceae & & Climber \\
\hline 43 & Ipomoea obscura (Linn.) Ker.-Gawl. & Convolvulaceae & Vadfudardi & Climber \\
\hline 44 & Ipomoea pes-tigridis Linn. & Convolvulaceae & Photial, Wagpadi & Climber \\
\hline 45 & Alangium salvifolium (L.) Wangerin & Cornaceae & Ankol & Tree \\
\hline 46 & Coccinea grandis ( Linn.) Voight. & Cucurbitaceae & Tindoli & Climber \\
\hline 47 & Luffa aegyptiacaMill. & Cucurbitaceae & Galku & Climber \\
\hline 48 & Momordica charantia Linn. & Cucurbitaceae & Karela & Climber \\
\hline
\end{tabular}


Arpit D. Gamit and Bharat B. Maitreya

\begin{tabular}{|c|c|c|c|c|}
\hline Sr No & Scientific Name & Family & Common Name & Habit \\
\hline 49 & Cyperus esculentus Linn. & Cyperaceae & & Herb \\
\hline 50 & Cyperus iriaL. & Cyperaceae & & Herb \\
\hline 51 & Cyperus rotundus Linn. & Cyperaceae & Chido & Herb \\
\hline 52 & Dillenia pentagyna Robx. & Dilliniaceae & Karmal & Tree \\
\hline 53 & Dioscorea bulbifera Linn. & Dioscoreaceae & & Climber \\
\hline 54 & Dioscorea hispidaDennst. & Dioscoreaceae & & Climber \\
\hline 55 & Dioscorea pentaphylla Linn. & Dioscoreaceae & & Climber \\
\hline 56 & Diospyros melanoxylon Robx. & Ebenaceae & Timbru & Tree \\
\hline 57 & Cordia dichotomaForst.f. & Ehretiaceae & Gundo & Tree \\
\hline 58 & Acalypha ciliataForsk. & Euphorbiaceae & Dadari & Herb \\
\hline 59 & Acalypha indica Linn. & Euphorbiaceae & Dadari & Herb \\
\hline 60 & Bridelia spinosa Willd. & Euphorbiaceae & Asan & Tree \\
\hline 61 & Euphorbia hirta Linn. & Euphorbiaceae & & Herb \\
\hline 62 & Jatropha gossypiifolia Linn. & Euphorbiaceae & & Shrub \\
\hline 63 & Phyllanthus emblica Linn. & Euphorbiaceae & Aavla & Tree \\
\hline 64 & Phyllanthus erecta (Medic.) Almeida & Euphorbiaceae & Bhonyaamli & Herb \\
\hline 65 & Phyllanthus virgatusForst. & Euphorbiaceae & Moti bhonyamli & Herb \\
\hline 66 & Abrus precatorius Linn. & Fabaceae & Chanothi & Climber \\
\hline 67 & Butea monosperma (Lamk.) Taub. & Fabaceae & Khakhro, Kesudo & Tree \\
\hline 68 & Cajanus cajan ( Linn.) Millsp. & Fabaceae & Tuver & Shrub \\
\hline 69 & Crotalaria juncea Linn. & Fabaceae & Shaniyu & Shrub \\
\hline 70 & Dalbergia sissoo DC. & Fabaceae & & Tree \\
\hline 71 & Dalbergia latifoliaRobx. & Fabaceae & Sisam & Tree \\
\hline 72 & Desmodium triflorum ( Linn.) DC. & Fabaceae & & Herb \\
\hline 73 & Erythrina variegata Linn. & Fabaceae & Pangaro & Tree \\
\hline 74 & Gliricidia sepium (Jacq.) H.B.K. & Fabaceae & Undir-mar & Tree \\
\hline 75 & Mucuna pruriens ( Linn.) DC. & Fabaceae & khajkhujali & Climber \\
\hline 76 & Ougenia oojeinensis (Robx.) Hochrest & Fabaceae & Tanas & Tree \\
\hline 77 & Pongamia pinnata (Linn.) Pierre & Fabaceae & Karanj & Tree \\
\hline 78 & Pterocarpus marsupium Robx. & Fabaceae & Biyo & Tree \\
\hline 79 & Tephrosia purpurea (Linn.) Pers. & Fabaceae & Sharpankha & Herb \\
\hline 80 & Flacourtia indica (Burm.f.) Merr. & Flacourtiaceae & Gargugal & Tree \\
\hline 81 & Enicostemma verticillare (Retz.) Baill. & Gentianaceae & Mamejevo & Herb \\
\hline 82 & Curculigo orchioidesGaertn. & Hypoxidaceae & Kali-musli & Herb \\
\hline 83 & Hyptissu aveolens ( Linn.) Poit. & Lamiaceae & & Herb \\
\hline 84 & Ocimum americanum Linn. & Lamiaceae & Ran tvulasi & Herb \\
\hline 85 & Ocimum tenuiflorum Linn. & Lamiaceae & Tulsi & Shrub \\
\hline 86 & Careya arboreaRobx. & Lecythidaceae & Kumbh & Tree \\
\hline 87 & Leea asiatica ( Linn.) Ridsd. & Leeaceae & Dindi & Shrub \\
\hline 88 & Asparagus racemosusWilld. & Liliaceae & Shatavari & Shrub \\
\hline 89 & Gloriosa superba Linn. & Liliaceae & & Climber \\
\hline 90 & Lindernia crustacea (L.) F. Muell. & Linderniaceae & & Herb \\
\hline 91 & Dendrophthoe falcata (Linn. f.) Ettingsh. & Loranthaceae & Vando & Shrub \\
\hline 92 & Lagerstroemia lanceolataWall. ex Wt. & Lythraceae & Nana bhondara & Tree \\
\hline 93 & Lagerstroemia parviflora Robx. & Lythraceae & Bhondaro & Tree \\
\hline 94 & Azanza lampas (Cav.) Alef. & Malvaceae & Ran-Behndi & Shrub \\
\hline 95 & Firmiana colorata (Robx.) R.Br. & Malvaceae & Kaushi & Tree \\
\hline 96 & Hibiscus rosa-sinensis Linn. & Malvaceae & Jasud & Shrub \\
\hline 97 & Kydia calycinaRobx. & Malvaceae & Warang & Tree \\
\hline
\end{tabular}


Plant diversity of kansari mavli sacred grove of songadh forest range in Tapi district, Gujarat, India

\begin{tabular}{|c|c|c|c|c|}
\hline Sr No & Scientific Name & Family & Common Name & Habit \\
\hline 98 & Sida acutaBurm. $f$. & Malvaceae & & Herb \\
\hline 99 & Sida cordata (Burm.f.) Boiss & Malvaceae & Bhoyabala & Herb \\
\hline 100 & Urena lobata Linn. & Malvaceae & Vagadau & Herb \\
\hline 101 & Martynia annua Linn. & Martyniaceae & Vinchhudo & Herb \\
\hline 102 & Soymida fabrifuga (Robx.) A. Juss. & Meliaceae & Royan & Tree \\
\hline 103 & Cissampelos pareira Linn. & Menispermaceae & Venivel & Climber \\
\hline 104 & Cocculus hirsutus (Linn.) Theob. & Menispermaceae & Vasanvel & Climber \\
\hline 105 & Tinospora cordifolia (Willd.) Miers ex & Menispermaceae & Galo & Climber \\
\hline 106 & Acacia senegal (L.) Willd. & Mimosaceae & Gorad & Tree \\
\hline 107 & Acacia tortilis (Forrsk.) Hayne & Mimosaceae & & Tree \\
\hline 108 & Acacia auriculaeformis Benth. & Mimosaceae & & Tree \\
\hline 109 & Albizia lebbeck (Linn.) Benth. & Mimosaceae & Shirish & Tree \\
\hline 110 & Albizia procera (Robx.) Benth. & Mimosaceae & Kilai & Tree \\
\hline 111 & Vachellia chundra (Robx.) & Mimosaceae & Khair & Tree \\
\hline 112 & Vachellia nilotica ( Linn.) P. J. H. Hurter & Mimosaceae & Bavval & Tree \\
\hline 113 & Ficus arnottiana (Miq.) Miq. & Moraceae & Khadakpayar & Tree \\
\hline 114 & Ficus hispida Linn.f. & Moraceae & DhedhUmbaro & Tree \\
\hline 115 & Ficus racemosa Linn. & Moraceae & Umbaro & Tree \\
\hline 116 & Musa paradisiaca Linn. & Musaceae & Kela & Herb \\
\hline 117 & Syzygium cumini (Linn.) Skeels & Myrtaceae & Jambu & Tree \\
\hline 118 & Boerhavia diffusa Linn. & Nyctaginaceae & & Herb \\
\hline 119 & Aeginetia indicaL. & Orobanchaceae & Ghost Flower & Herb \\
\hline 120 & Biophytum sensitivum (Linn.) DC. & Oxalidaceae & Zarero & Herb \\
\hline 121 & Argemone mexicana Linn. & Papaveraceae & Darudi, & Herb \\
\hline 122 & Cryptolepisdubia (Burm.f.) Almeida & Periplocaceae & & Climber \\
\hline 123 & Plumbago zeylanica Linn. & Plumbaginaceae & Chitrak & Shrub \\
\hline 124 & Cynodon dactylon ( Linn.) Pers. & Poaceae & Darbh & Herb \\
\hline 125 & Dendrocalamus strictus (Robx.) Nees & Poaceae & Narvans & Herb \\
\hline 126 & Oplismenus compositus (L.) P.Beauv. & Poaceae & & Herb \\
\hline 127 & Oryza sativa Linn. & Poaceae & Bhat, Chokha & Herb \\
\hline 128 & Zea mays Linn. & Poaceae & Makai & Herb \\
\hline 129 & Ventilago denticulataWilld. & Rhamnaceae & Asi, Asvel & Climber \\
\hline 130 & Ziziphus jujubaMill. & Rhamnaceae & Bor & Tree \\
\hline 131 & Haldina cordifolia (Robx.) Ridsd. & Rubiaceae & Haldu & Tree \\
\hline 132 & Hymenodictyon orixense (Robx.) Mabb. & Rubiaceae & Alipagi & Tree \\
\hline 133 & Mitragyna parvifolia (Robx.) Korth. & Rubiaceae & Kalam & Tree \\
\hline 134 & Morinda pubescensSm. & Rubiaceae & Ali & Tree \\
\hline 135 & Aegle marmelos ( Linn.) Correa & Rutaceae & Bili & Tree \\
\hline 136 & Schleicheraoleosa (Lour.) Oken & Sapindaceae & Kusum & Tree \\
\hline 137 & Madhuca indicaGmelin & Sapotaceae & Mahudo & Tree \\
\hline 138 & Datura metel Linn. & Solanaceae & Daturo & Herb \\
\hline 139 & Physalis minima Linn. & Solanaceae & Popti & Herb \\
\hline 140 & Melochia corchorifoliaL. & Sterculiaceae & & Herb \\
\hline 141 & Sterculia urensRobx. & Sterculiaceae & Kadio & Tree \\
\hline 142 & Tacca leontopetaloides (Linn.) Kuntze & Taccaceae & & Shrub \\
\hline 143 & Grewia tiliaefoliaVahl & Tiliaceae & Dhaman & Tree \\
\hline 144 & Triumfetta pentandraA. Rich. & Tiliaceae & Zipti & Herb \\
\hline 145 & Holoptelea integrifolia (Robx.) Planch. & Ulmaceae & Kanjo, Papda & Tree \\
\hline 146 & Gmelina arboreaRobx. & Verbenaceae & Sevan & Tree \\
\hline
\end{tabular}


Arpit D. Gamit and Bharat B. Maitreya

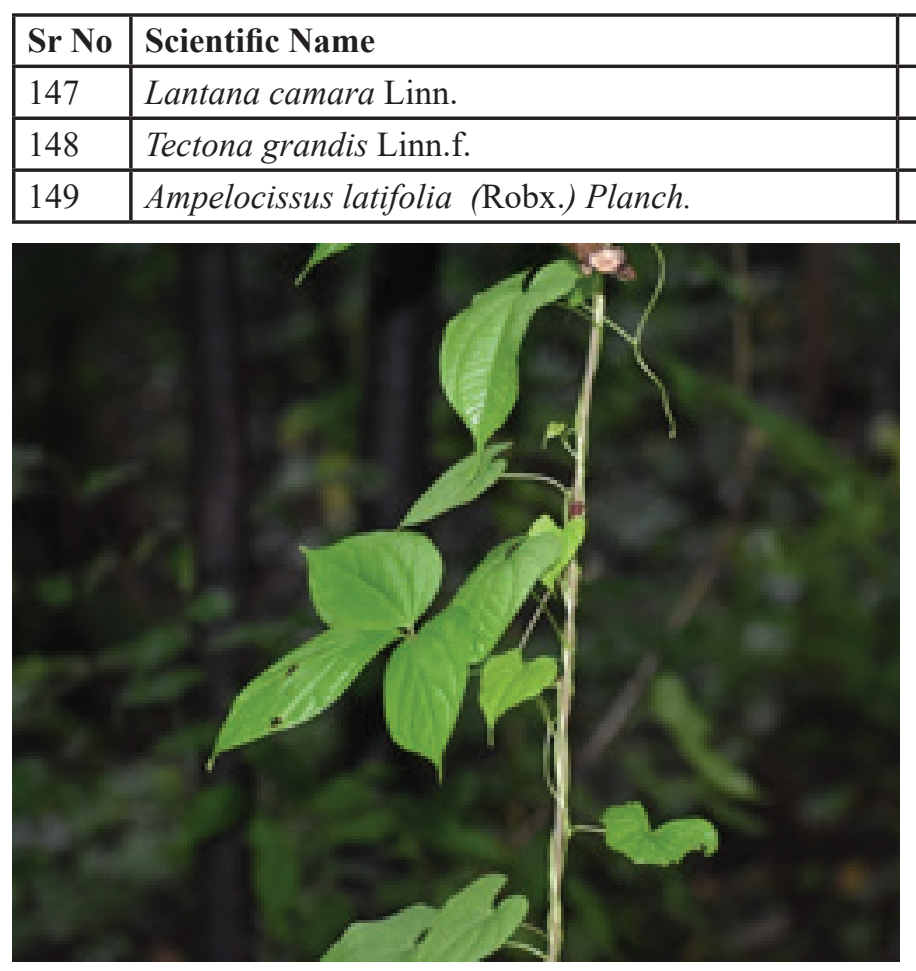

Dioscorea hispida Dennst

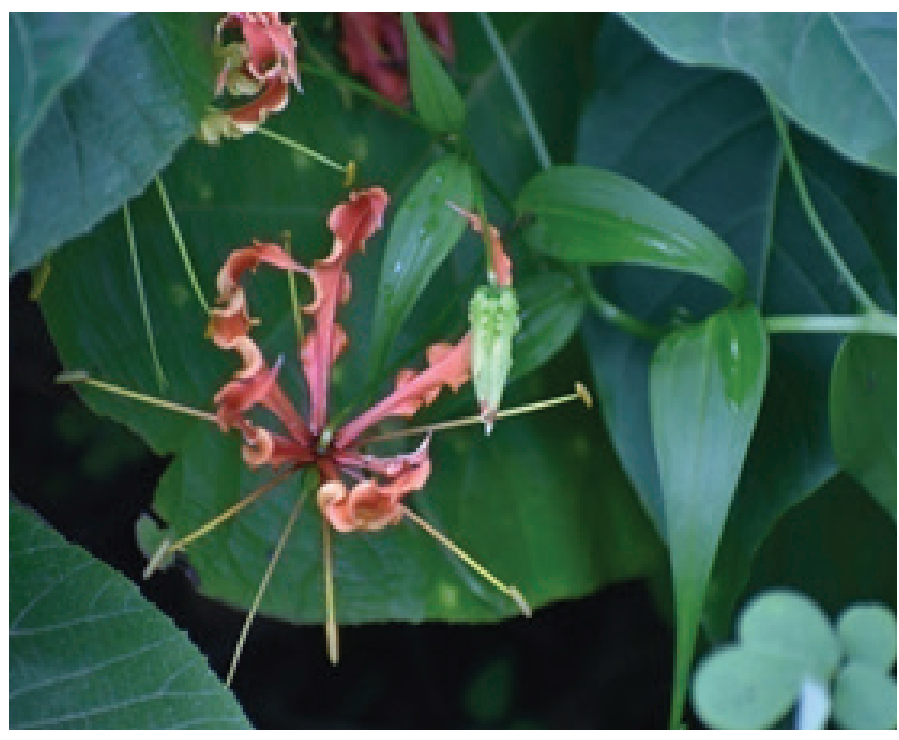

Gloriosa superba Linn.

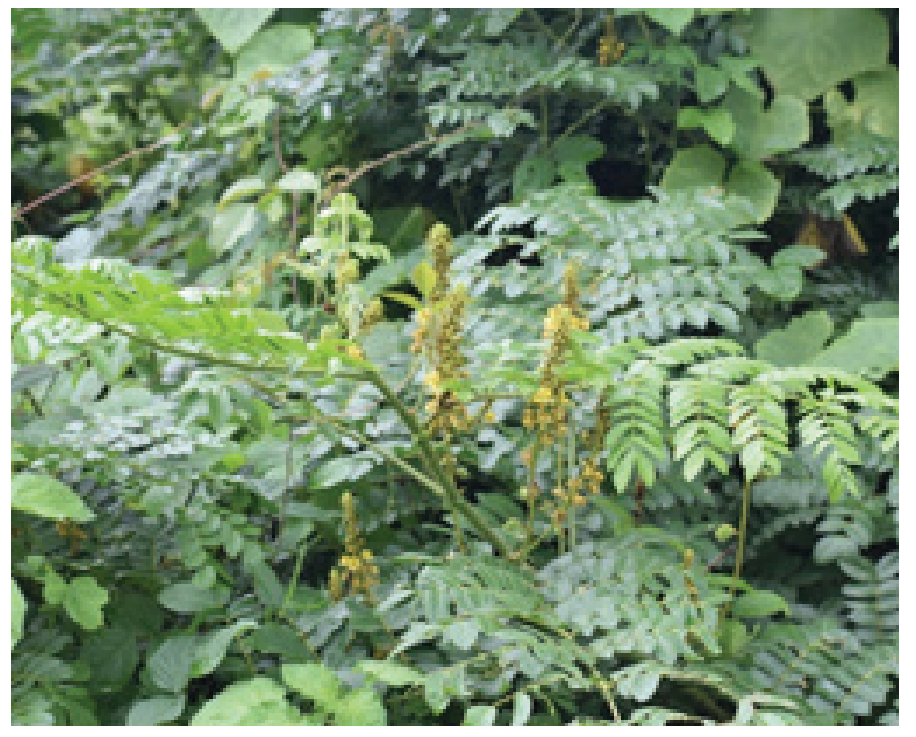

Caesalpinia bonduc (L.)Roxb

\begin{tabular}{|c|c|c|}
\hline Family & Common Name & Habit \\
\hline Verbenaceae & & Shrub \\
\hline Verbenaceae & Sag & Tree \\
\hline Vitaceae & JangaliDraksha & Climber \\
\hline
\end{tabular}

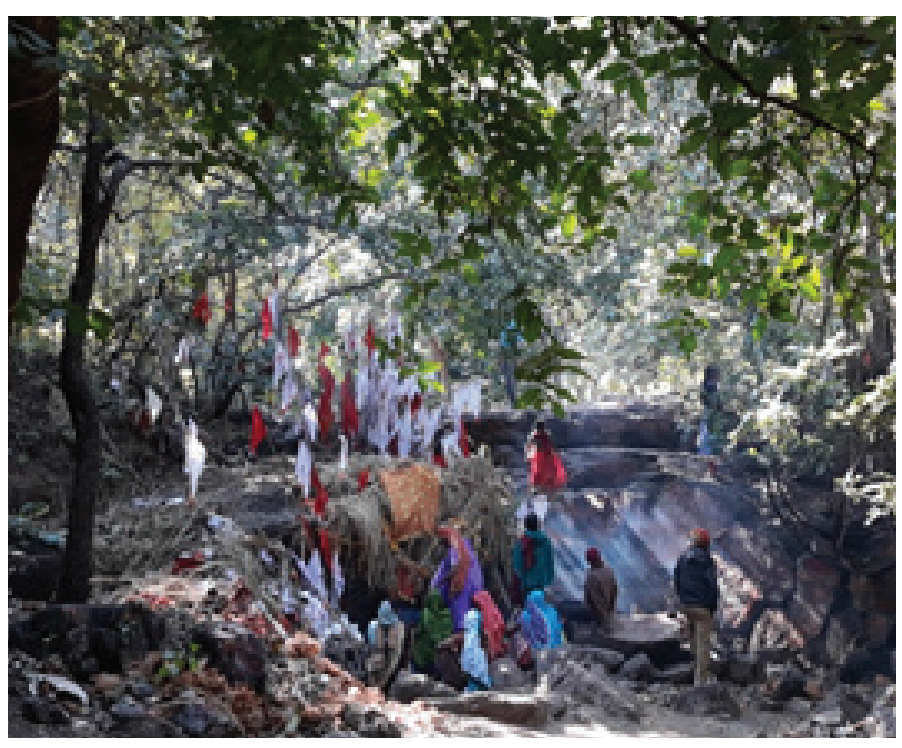

Post Monsoon ceremony

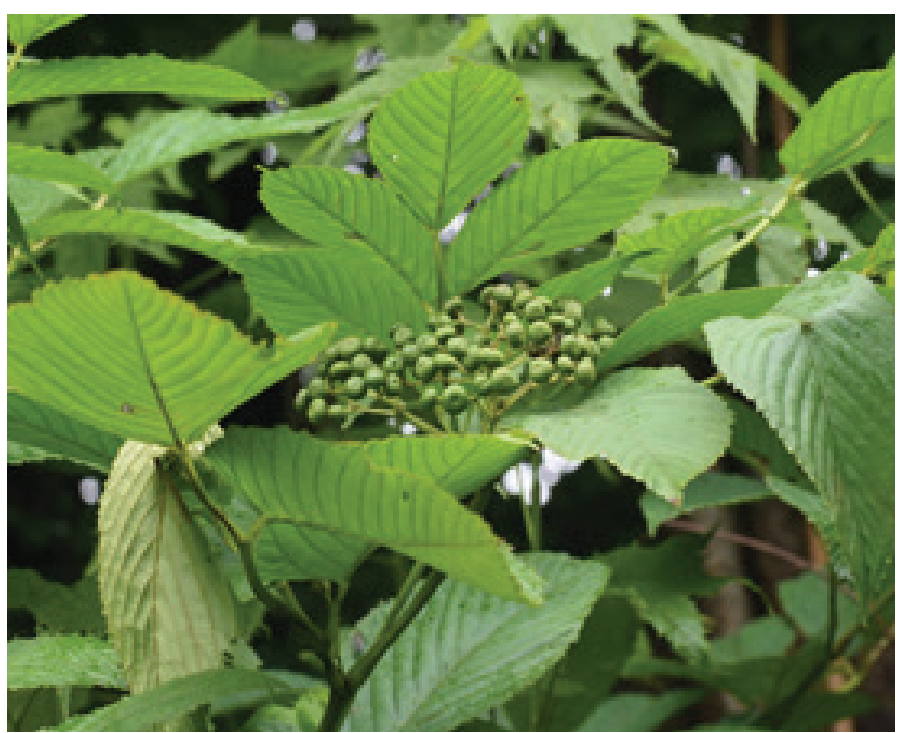

Leea asiatica ( Linn.) Ridsd.

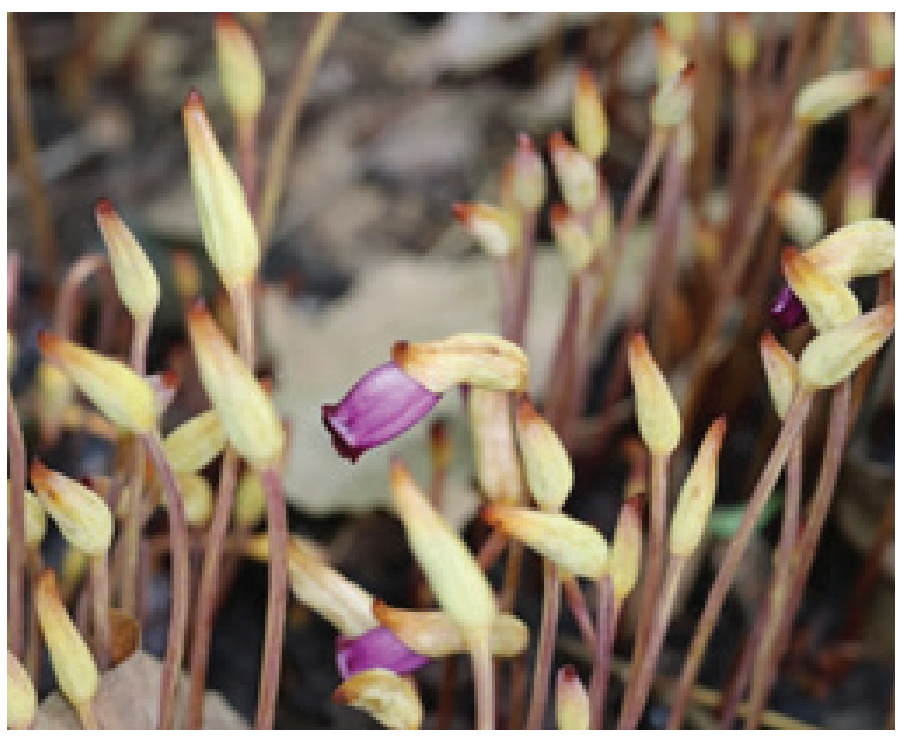

Aeginetiai ndica L. 


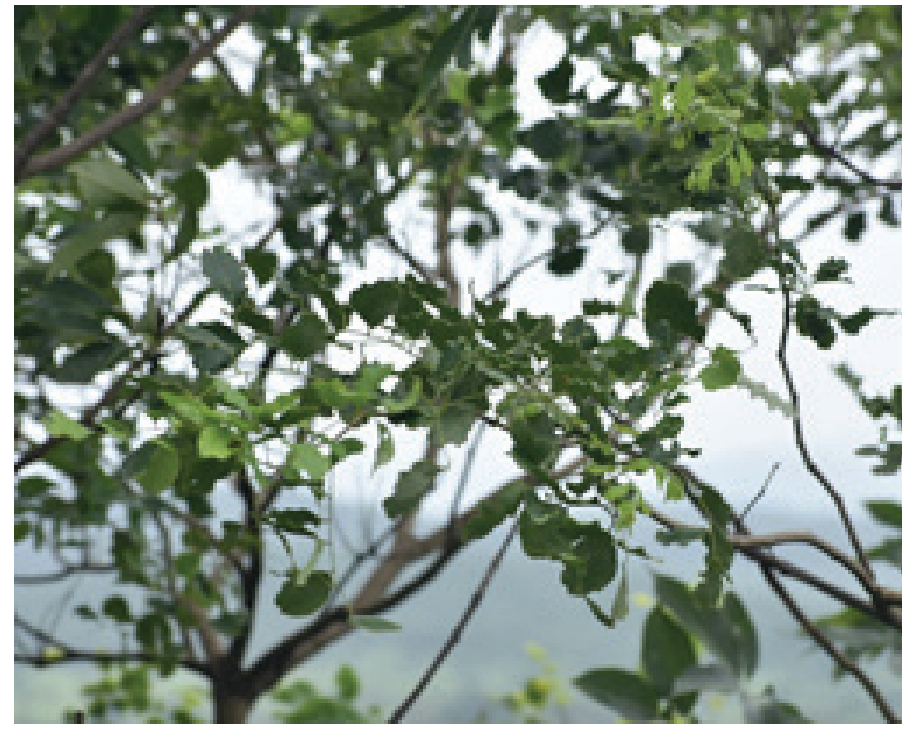

Dalbergia latifolia Robx.

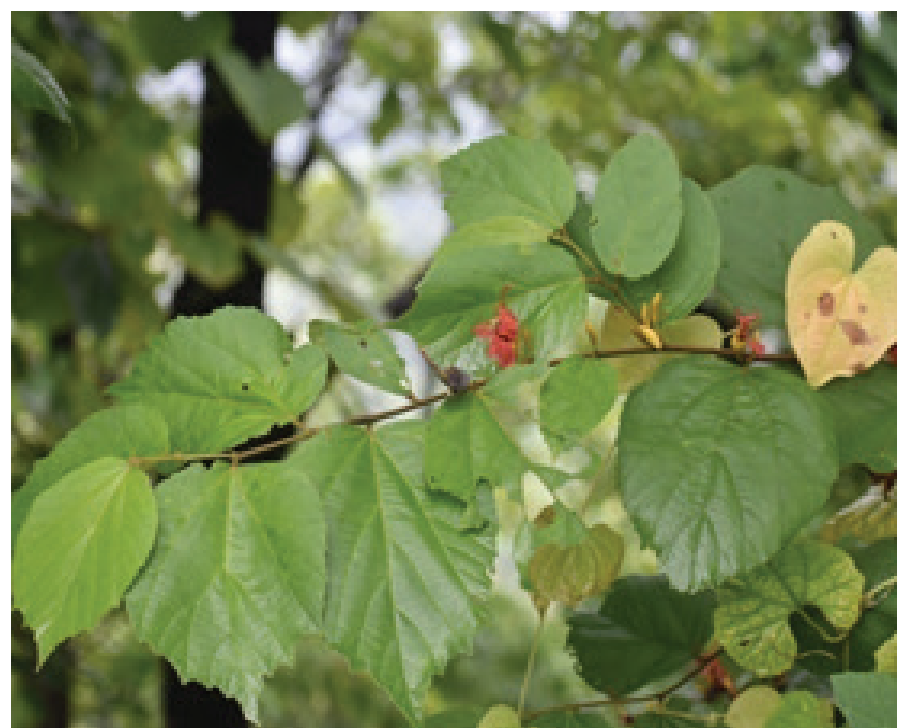

Helicteres isora Linn.

REFERENCES

BhakatR.K. (1990) Tribal ethics of Forests conservation. Yojana (March 16-31):23-27.

BrandisD (1987b) Indigenous Indian Forestry: sacred Groves in Indian Forestry, Working Oriental Institute, UK.pp. 12-13.

Burman R.J.J. 1992. The institution of sacred grove. J. Indian Anthropol. Soc. 27: 219-238

Gadgil M. and Vartak V.D. (1975) Sacred groves of India - a plea of the continuous conservation. J. Bombay Nat. Hist. Soc. 72 (2): 313-320.

Gadgil M. and Vartak V.D. (1976) Sacred groves of Western Ghats of India. Economic Botany; 30:152-160.

Haridasan K. and Rao R.R. (1985)Forest .ora of Meghalaya, Vol. 1. Bishen Singh and Mahendrapal Singh, Dehra Dun. 1985.

JamirS.A., and Pandey H.N., (2003) Vascular plant diversity in sacred groves Jaintia Hills in northeast India. Biodiversity and conservation 12: 1497- 1510.

JamirS.A.,Upadhaya K. and Pandey H.N., (2006) Life form compostion and stratification of montane humid forests in Meghalaya, northeast India. Tropical Ecology 47 (2) 183-

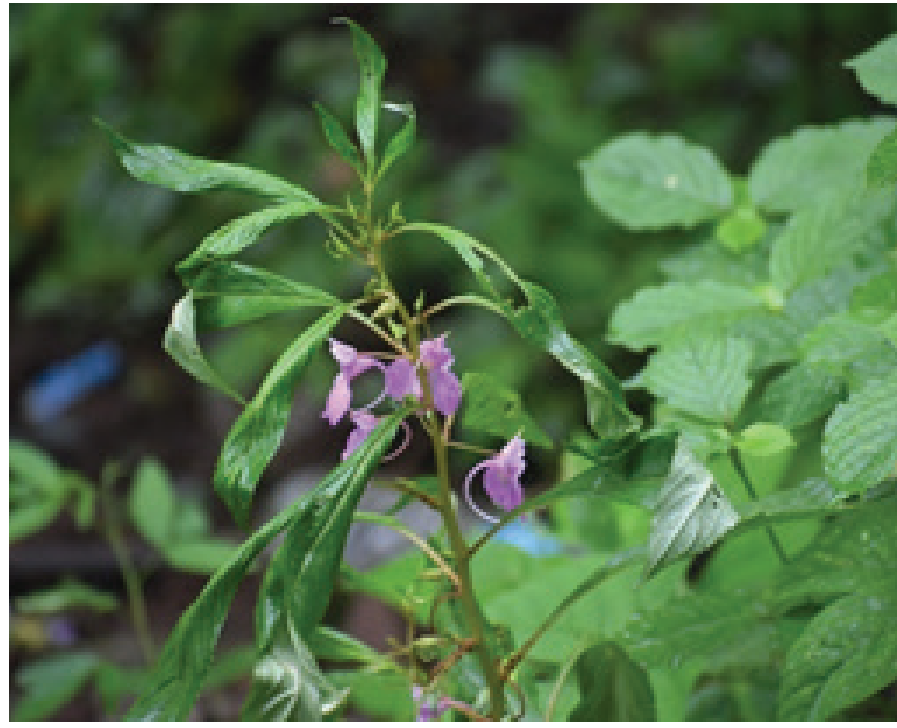

Impatiens balsamina Linn.

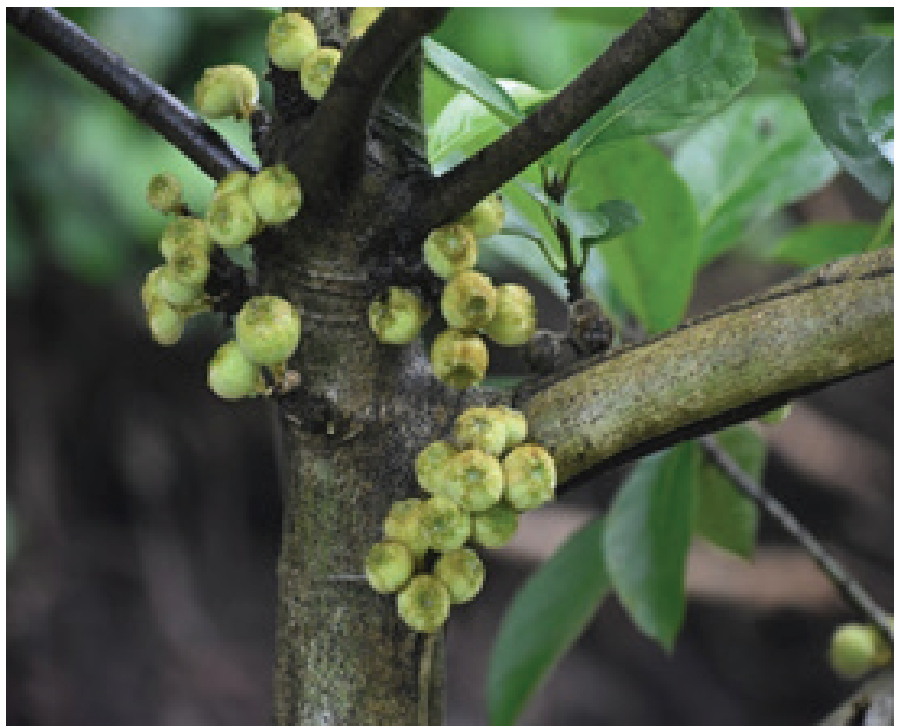

Ficus hispida Linn.f.

190.

Khan M.L., Menon S. and Bawa K.S. (1997)E.ectiveness of the protected area networkin bio-diversity conservation: a case study of Meghalaya state. Biodiv. Conserv. 6: 853868.

Maru, R.N. and Patel R.S., (2013) Ethno-Botanical Survey of Sacred Groves and Sacred Plants of Jhalod and Surrounding Areas in Dahod District, Gujarat, India, Res.J.Recent.Sci. Vol. 2 (ISC-2012), 130-135.

Mehta P.K., and Jain B.K., (2011)Ethnobotanical study of Sacred groves of Poshina forest of Sabarkanth district, North Gujarat, Int, J. Plant Sci., 6 (2), 362-366

Nimisha, T., Singh R.S., Bakhori. B., Dalal C., Parmar D., and Mishra B. (2013) "The world's only inland mangrove in sacred grove of Kachchh, India, is at risk", Current Science, Vol. 105 (8)

Patel H.R. and Patel R., (2013) Ethnobotanical Plants Used by the Tribes of R.D.F.Phoshina Forest Range of SabarkanthaDistrict,North Gujarat, India, Int. J.Sci. Res. Publication, 3 (2), 1-8

Patel P.K. and Patel M.K., (2013) Sacred groves in conservation of plant biodiversity in Banaskantha district, Gujarat, 
India. 5 (1) 13-16.

Patel R., Mahato A.K., and Patel Y.S., (2014)“Study on the Floristic Diversity of Two Newly Recorded Sacred Groves from Kachchh District of Gujarat, India", Indian Journal of Plant Sciences, 3 (1), 75-81
Ramakrishan P.S. (1996) Conserving the sacred: from species to Landscapes. Nature Resour. UNESCO 32: 11-19.

Vartak V.D. and Gadgil M. (1973) DevRahati: an ethnobotanical study of the forests preserved on grounds of religious beliefs. Abstract, Proc. Indian Sci. Cong. 60: 341. 